\title{
Prolonging the Network Lifetime using Fuzzy logic in Wireless Sensor Network
}

\author{
Palkin Sharma \\ Student \\ Department of Electronics and Communication \\ Punjabi University, Patiala, \\ Punjab, INDIA
}

\author{
Sonia Goyal \\ Assistant Professor \\ Department of Electronics and Communication \\ Punjabi University, Patiala, \\ Punjab, INDIA
}

\begin{abstract}
Wireless sensor networks include deployment of large number of sensor nodes to sense, process and communicate the data to the sink. The small sized sensor nodes are cheap and can be easily deployed in harsh physical environments but the low battery source for the nodes act as a major limitation for the sensor networks to operate efficiently. The protocols have been proposed in recent times to make optimum use of the energy. Power Efficient Gathering in Sensor Information System (PEGASIS) has been considered as an efficient approach as compared to the Low Energy Adaptive Clustering Hierarchy (LEACH). In this paper, a new approach for selecting the leader of the chain has been proposed using Fuzzy Logic in which residual energy and proximity to the base station has been taken as two parameters and combining these two parameters the leader is selected which aggregates and communicates the data further to the sink. The simulation results have shown that this proposed work has improved the performance metrics of the network and thus extended the lifetime of network.
\end{abstract}

\section{General Terms}

Prolonged Network lifetime in Wireless Sensor Network

\section{Keywords}

Environment, Sensor nodes, lifetime, optimum

\section{INTRODUCTION}

The development of small sized sensors has extended the application fields of Wireless Sensor Networks. Wireless sensor network deploys large number of sensors that can sense the physical environment, process the collected data and ten send it to the user. Small sized sensors perform these tasks efficiently but these nodes have limited battery power supply. As these tasks consume much of energy, nodes start dying and eventually network operation stops when all the nodes die. Various hierarchical protocols proposed the idea of clustering which proved to be an efficient technique for increasing the lifetime of networks as compared to directly sending the data to base station. The energy required to send the data packets of length $\mathrm{L}$ to distance $d$ (base station) is given as:

$$
\begin{aligned}
\mathrm{E} T_{x} & =\mathrm{L} * E_{\text {elec }}+\mathrm{L} * \epsilon_{f s} * d^{2} \text { if } \mathrm{d}<<d_{o} \\
& =\mathrm{L} * E_{\text {elec }}+\mathrm{L} * \epsilon_{m p} * d^{4} \text { if } \mathrm{d}>>d_{o}
\end{aligned}
$$

The amount of energy required to receive a packet of $L$ bits is given by:

$$
\mathrm{E} R_{x}=\mathrm{L} * E_{\text {elec }}
$$

where $E_{\text {elec }}$ is the energy being dissipated to run the transmitter or receiver circuitry, $\epsilon_{f s}$ and $\epsilon_{m p}$ is the amount of energy dissipated per bit, $d_{o}$ is threshold distance [1]. Low Energy Adaptive Clustering Hierarchy (LEACH) [2] is the first ever clustering based protocol in which nodes are divided into clusters and for each cluster, a cluster head is selected randomly. LEACH provided the energy load balancing between the nodes by rotating the cluster heads. This approach increased the efficiency of the network. From then on, many researches focussed on the various efficient ways of cluster formation and selection of the cluster heads so that operation of the network can be extended for a longer time. Other protocols like Stable Election Protocol (SEP) [3] divided the nodes on the basis of energy levels and nodes with higher residual energy are prioritized for the selection of cluster heads. This approach also improved the performance of the network in terms of increasing the lifetime. Various improvements of LEACH protocol has also been proposed that choose different criteria for the selection process of cluster head. PEGASIS [4] is chain based protocol in which nodes do not directly communicate with the base station, instead nodes connect with the nearest neighbour and thus start forming chain. In PEGASIS, randomly a node is chosen that becomes leader and that node will collect the data and send it to the sink. The performance of PEGASIS is much better than LEACH. The chain based protocol PEGASIS randomly selects a node to forward the data collected from the other nodes. The random selection will sometime lead to high delay due to formation of long links. So improvements are required in this area for the chain based protocols to perform better. In this paper, a new approach has been proposed for selecting the leader node in the chain of nodes using Fuzzy Inference System (FIS) that considered basically two parameters as residual energy and proximity to the Base Station (BS). Simulation shows that proposed work balances the energy consumption and leads to the extended network lifetime.

\section{RELATED WORK}

Various hierarchical protocols have been proposed from recent times in order to increase the network lifetime and also make the network efficient. Initially LEACH protocol divided the whole network into clusters and selected a node from each cluster, similarly PEGASIS formed chain of nodes for communication of data between the nodes. Further improvements led to the development of more and more protocols and each one of the protocol tried to improve the performance metrics of network in one or the other way. Hybrid Energy Efficient Distributed Clustering (HEED) [5] proposed a new method for selection of the Cluster head based on residual energy of the node and intra cluster communication cost. It balanced the energy load on the nodes and thus improved the performance of the network. The 
cluster heads closer to base station used to get die faster than other nodes in the previous approaches. This problem has been solved by the protocol Energy Efficient Unequal Clustering (EEUC) [6]. This approach reduced the size of clusters that are close to the base station as compared to clusters that are away from base station. In LEACH, cluster heads are selected randomly based on the probability, random selection of Cluster Heads $(\mathrm{CH})$ will not lead to optimum number of $\mathrm{CHs}$ in the network, so an Adaptive Cluster Habit $(\mathrm{ACH})$ [7] protocol solved this problem and thus increased the efficiency of network by $31.8 \%$ than the LEACH protocol. Fuzzy logic has been used as a method to select the cluster head in a cluster by considering and combining two or more parameters in [8]. The input parameters have been taken as noise factor, residual energy and distance. The performance of the network has improved and network lifetime also increased even in the noisy environment.

Analysis of the LEACH type schemes basically involves homogeneous environment in which all the nodes have same energy levels. In [9], heterogeneous sensor network has been considered and uses geographical position to provide scalability. Basically nodes forward data to the nearest node and due to shortened distance energy consumption also reduces. The proposed protocol is a geographical position based clustering algorithm. The nodes independently select themselves as cluster head by comparing their energy levels with other nodes. This method showed a great improvement as compared with LEACH, at the end of $100^{\text {th }}$ round , remaining energy in case of Leach is $0.9 \mathrm{~J}$ whereas in case of proposed protocol energy is $1.5 \mathrm{~J}$. So thus energy consumption is quite less. PEGASIS protocol improved the performance of the LEACH, further the modified version of PEGASIS is proposed in [10] where a different method for chain formation and leader selection has been suggested. The chain formation method allows visited nodes that are above in the ordering to be used again and again for the connections. The leader selection is also based on the energy level, distance and number of nodes by using a function depending on these three parameters. This allows the best node to be chosen for the data communication and routing purpose. The proposed work led to prolonged network lifetime by $40 \%$ as compared to the PEGASIS. In [11], Fuzzy logic has been proposed for the selection purpose of cluster head, the parameters used for the selection are energy, distance and density. The output parameter is the chance of a node to become a cluster head. The rule base is used for making the rules as per IF-THEN rule base evaluation in the Fuzzy systems. The results of the proposed work has been compared with the LEACH protocol, the number of alive nodes become zero at the $3200^{\text {th }}$ round for the proposed protocol, whereas all nodes die at $1800^{\text {th }}$ round in case of LEACH. Thus various approaches have been proposed for the energy efficiency in the wireless sensor networks in the recent works. In this paper, various points has been considered and a variant approach has been proposed. The proposed work improves the performance metrics of the network along with the prolonged network lifetime which is the major concern of this proposed protocol. In [12], an Intelligent Cluster Routing proposed an improved approach over SEP by choosing the fittest node as cluster head in terms of maximum energy. This approach is applied on heterogeneous network and the results showed a great improvement than SEP as the round at which all nodes die in case of proposed protocol is 2600, whereas in case of SEP, nodes die at $1800^{\text {th }}$ round. So this protocol improved the initial approach.

\section{PROPOSED WORK}

The purpose of the proposed work is to increase the network lifetime and to improve the performance of the sensor network. The proposed model involves the randomly deployment of sensors, chain construction, leader selection and then data transmission.

The leader selection method leads to improvement of the energy consumption of the network. The leader is selected using the fuzzy inference system in which input parameters are residual energy and proximity to base station. The deployment phase requires initialising some parameters like node initial energy, energy dissipation, packet size. The randomly deployed network is shown in Figure 1.The table shows the network parameters assumed initially so as to carry out the simulations. The simulations are carried out in MATLAB. The distance is calculated between the nodes and from nodes to the sink. Both these distances are calculated for each of the node and then the farthest node is determined based on the distance calculations. Thus bubble sort is applied to obtain the farthest node from the sink and it is labelled as first node. In this way nodes are sorted in descending fashion and then chain is constructed by connecting each node with nearest possible node till all the nodes get connected. After the formation of chain, as shown in Figure 2, the leader selection phase starts, leader is selected with the help of Fuzzy Inference System. For this Mamdani system is used that contains three parts: fuzzifier, inference engine and defuzzifier [13].

The MATLAB consists of Fuzzy toolbox in which two parameters have been used as input variables : residual energy and proximity to base station as shown in Figure 3. These variables are divided into five levels : low, medium, high, very high, very low are used for residual energy whereas near, very near, medium, far, very far are used for the proximity to base station. Since two parameters are divided into five levels so 25 chance values are possible and thus 25 rules are possible for evaluation as shown in Figure 4.

In every round, the chance to become leader is calculated using IF-THEN rules and the one having the highest chance is selected as the leader which further leads to data transmission phase in which node collect the data from all the other nodes and transmit it to the base station.

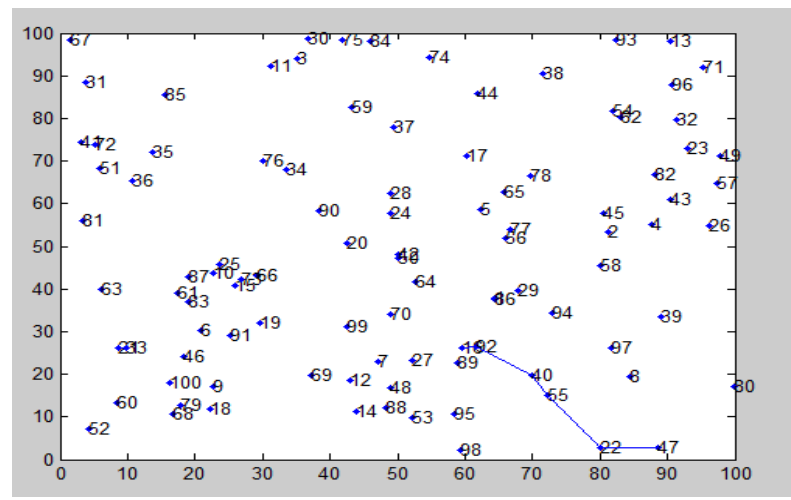

Figure 1. The randomly deployed nodes 


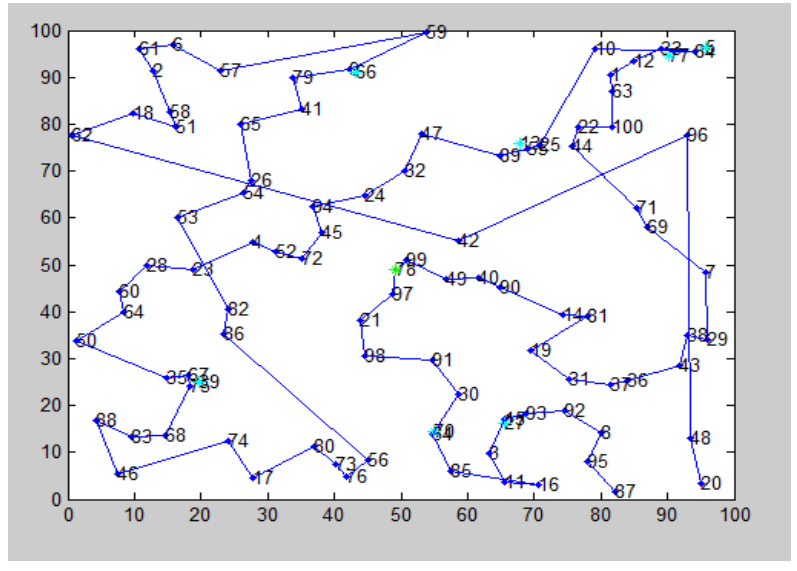

Figure 2. The chain construction among the nodes

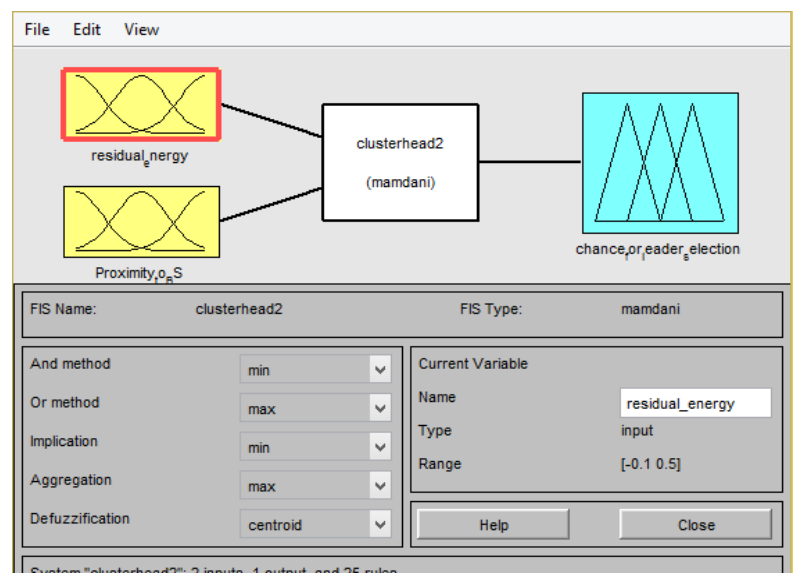

Figure 3. Fuzzy Inference System toolbox in MATLAB

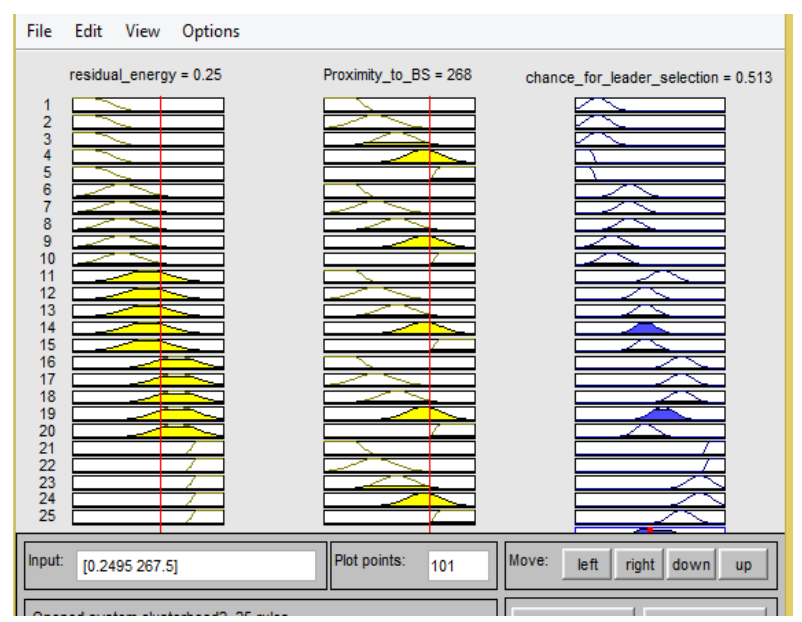

Figure 4. Rule based evaluation using IF-THEN rule

The steps of the proposed method are as follows:

1. Initialize number of nodes, energy and location to the base station.

2. Calculate the distances between the nodes and the sink.

3. Chain Construction starts by finding the farthest node form the BS

4. Join the farthest node to the minimum distance alive node and construct a chain.

5. Leader selection using Fuzzy logic.
6. Data transmission by aggregating the data from all nodes and then sending it to the sink.

7. Continue till the lifetime is over i.e. when all the nodes run out of battery power.

\section{SIMULATION AND RESULTS}

The simulation is carried out in the MATLAB. The fuzzy toolbox enabled the fuzzy logic calculations in the simulator itself. For the network to run properly, some conditions are to be pre assumed and values are given initially. Some of the parameters are initialised as shown in the Table 1.

\section{Table 1. Simulation Parameters}

\begin{tabular}{|c|c|}
\hline Parameters & Value \\
\hline $\begin{array}{c}\text { Network } \\
\text { Area }\end{array}$ & $100 * 100$ \\
\hline $\begin{array}{c}\text { No. of } \\
\text { nodes }\end{array}$ & 100 \\
\hline $\begin{array}{c}\text { Initial } \\
\text { energy }\end{array}$ & $0.5 \mathrm{~J}$ \\
\hline $\mathrm{E}_{\mathrm{amp}}$ & $0.0013^{*} 0.000000000001$ \\
\hline $\mathrm{E}_{\mathrm{fs}}$ & $10^{*} 0.000000000001$ \\
\hline $\begin{array}{c}\text { Transmit } \\
\text { packet size }\end{array}$ & 2000 \\
\hline $\mathrm{E}_{\text {fusion }}$ & $5 * 0.000000001$ \\
\hline
\end{tabular}

The simulations are carried out for 100 nodes initially, firstly for the chain based protocol for which nodes die completely till 2200 rounds, and for the proposed protocol the rounds are around 4500. As shown in the Figure 5, x-axis shows number of rounds and y-axis shows the number of alive nodes.

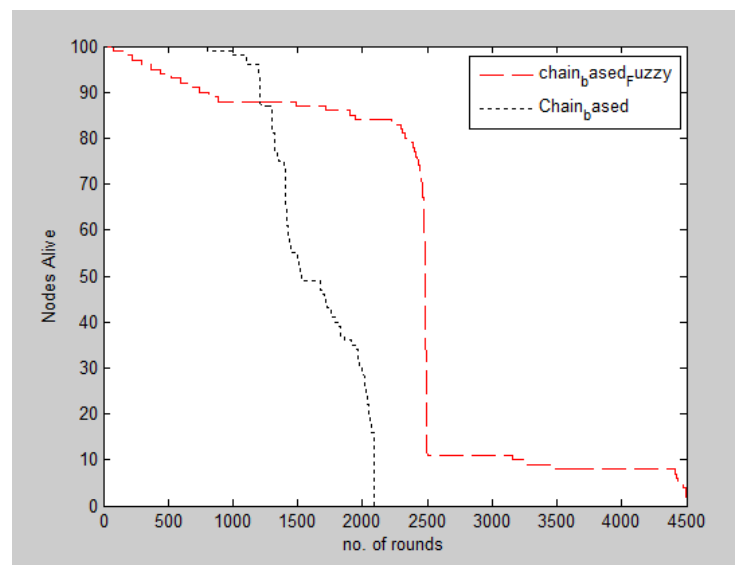

Figure 5. Comparison of the number of alive nodes as the rounds continues. 


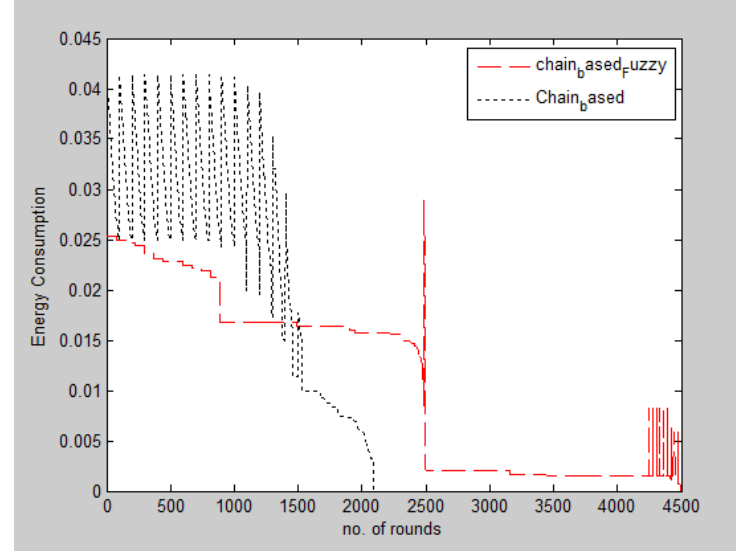

Figure 6. Comparison showing energy consumption with increasing number of rounds

The simulation parameters have been listed in the Table 1 which is the pre requisite for carrying out the simulations in MATLAB. Figure 6 shows the amount of energy consumed for the chain based protocol and the proposed chain based fuzzy protocol, where $\mathrm{x}$-axis shows the number of rounds and $y$-axis shows the energy consumption in Joules. The energy consumption is also reduced for the proposed protocol and thus led to prolonged network lifetime.

Table 2. Network performance comparison in terms of rounds till all nodes die

\begin{tabular}{|l|l|l|}
\hline Energy (J/node) & \multicolumn{1}{|c|}{ Protocol } & $\begin{array}{c}\text { Round when all } \\
\text { nodes die }\end{array}$ \\
\hline 0.5 & PEGASIS [4] & 1544 \\
\hline 0.5 & SCBC [13] & Around 2000 \\
\hline 0.5 & Proposed & Around 4500 \\
\hline
\end{tabular}

Table 2 shows the comparison in terms of number of rounds till all of the nodes die in a network. According to [4], all the nodes die at the end of $1544^{\text {th }}$ round and in the similar Sector Chain Based Clustering (SCBC) [14] approach the round at which all nodes die is around 2000 whereas in the proposed approach, nodes die at the end of around $4500^{\text {th }}$ round. So the proposed approach performs better than the PEGASIS and SCBC protocol.

\section{CONCLUSION}

As per the results of simulation, the proposed algorithm shown a significant improvement in terms of increasing the energy efficiency of the network. The combination of parameters for the selection of leader among the nodes prolonged the network lifetime and also reduced the energy consumption. The proposed protocol performs better than the PEGASIS and chain based protocol. The proposed work can be further extended for the multiple sinks in the network and performance can be evaluated.

\section{REFERENCES}

[1] W.B. Heinzelman, A.P Chandrakasan, H.Balakrishnan, “ An Application Specific protocol Architecture for Wireless Microsensor Networks", IEEE Transactions $n$ Wireless Communication, Vol. 1, pp. 660-670, 2002

[2] Z.Yang, J.Liu and X.Chen, "An Optimal Mechanism of LEACH protocol for Wireless Sensor Network", International Colloquim on Computing Communication,
Control and Management, Sanya, China, pp. 254-257, 2009.

[3] G.Smaragdakis, I. Matta and A. Bestavros, "SEP: Stable Election Protocol for clustered heterogeneous Wireless Sensor Networks", Deptt. Of Computer Science, Boston University, USA, Tech. Rep. BUCS-TR, 2004

[4] S. Lindsey and C.S. Raghvendra, " PEGASIS: Power Efficient Gathering in Sensor Information System”, IEEE Aerospace Conference Proceedings, pp 1125-1130, 2002

[5] O. Younis and S. Fahmy, "HEED: A Hybrid Energy Efficient Distributed Clustering Approach for Ad hoc Sensor Networks", IEEE Transactions on Mobile Computing, pp. 90-93, 2010

[6] Chengfa Li, Mao Ye, Guihai Chen, “ An Energy Efficient Unequal Clustering Mechanism for Wireless Sensor Networks", IEEE Mobile Adhoc and Sensor Systems Conference, pp. 604, 2005

[7] Ashfaq Ahmad, Nadeem Javaid, Zahoor Ali Khan, "Routing Scheme to Maximize Lifetime and Throughput of Wireless Sensor Networks", IEEE Sensors Journal, Vol. 14, No. 10, 2014

[8] Ravendra Singh, Itika Gupta, A.K Daniel, “ Position based Energy Efficient Clustering Protocol under Noisy Environment for sensor Networks using Fuzzy Logic Technique", IEEE Science and Information Conference, pp. 843-849, 2014

[9] M.S. Jahan, A.Sali, "Energy efficient geographical and power based clustering algorithm for heterogeneous wireless sensor networks", IEEE 17th Asia Pacific Conference on Communications, pp. 826-831, 2011

[10] Madhuri Gupta, Laxmi Saraswat, "Energy Aware Data Collection in Wireless Sensor Networks using Chain based PEGASIS", IEEE Recent Advances and Innovations in Engineering (ICRAIE), pp.1-5, 2014

[11] Oussama Ben Belghith, Lasaad Sbita, " Extending the Network Lifetime of Wireless Sensor Networks using Fuzzy Logic", 12th IEEE International Conference on Systems, Signals \& Devices (SSD), pp. 1-5, 2015

[12] Arun Kumar, Vijay Kumar Katiyar, “ Intelligent Cluster Routing: An Energy Efficient Approach for Routing in Wireless Sensor Networks", International Journal of Computer Applications, Vol. 110, No. 5, pp.18-21, 2014

[13] Z.W. Siew, A. Kiring, “ Energy Efficient Clustering Algorithm in Wireless Sensor Networks using Fuzzy Logic Control”, IEEE Colloquim on Humanities, Science and Engineering, pp. 392-397, 2011

[14] N. Tan, N.Viet, “ SCBC: Sector Chain Based Clustering Routing Protocol for Energy Efficiency in Heterogeneous Wireless Sensor Network", IEEE International Conference on Advanced Technologies for Communications, pp. 314- 319, 2015 